\title{
Las representaciones sociales como marco para comprender las respuestas humanas en el cuidado enfermero
}

\section{Social representations as a framework for understanding human} responses in nursing care

\section{Representações sociais como um quadro para a compreensão das respostas humanas nos cuidados de enfermagem}

\author{
Vanesa Jiménez Arroyo ${ }^{1}$, Yesica Yolanda Rangel Flores ${ }^{2}$ \\ ${ }^{1}$ Universidad Michoacana de San Nicolás de Hidalgo. Profesora Investigadora de Tiempo Completo. Estudiante del Doctorado \\ Interinstitucional en Enfermería. \\ ${ }^{2}$ Universidad Autónoma de San Luis Potosí, México. Licenciada y maestra en Enfermería, Dra. en Ciencias Sociales. Profesor \\ investigador de la Facultad de Enfermería de la UASLP. Línea de investigación: Género y salud sexual y reproductiva. \\ Cómo citar este artículo en edición digital: Jiménez Arroyo, V., \& Rangel Flores, Y.Y. (2017). Las representaciones sociales como \\ marco para comprender las respuestas humanas en el cuidado enfermero. Cultura de los Cuidados (Edición digital), 21(49). \\ Recuperado de http://dx.doi.org/10.14198/cuid.2017.49.16 \\ Correspondencia: Remitirse al correo electrónico. Correo electrónico: yrangelmaestria@hotmail.com \\ Recibido: 14/02/2017; Aceptado: 20/06/2017
}

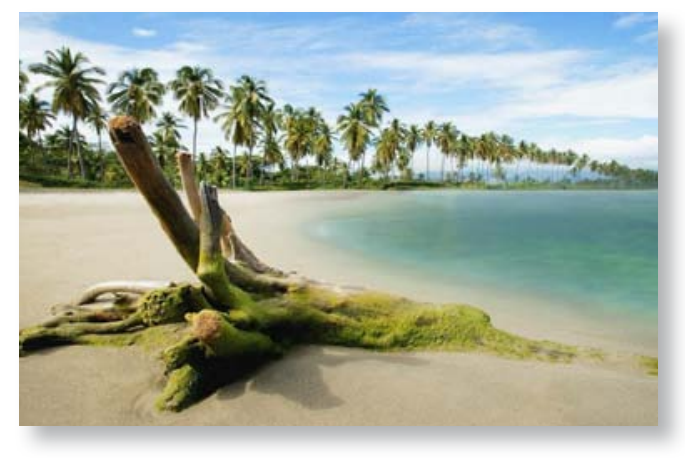

ABSTRACT

Human responses are complex and are based on how relevant social, cultural, economic and political micro, meso and macro in which people operate contexts conditions. The nurse needs an epistemological, philosophical and methodological perspective that allows him to understand the ways in which people are positioned against health-disease processes depend not only on a personal decision but a posture collectively built. The aim is to reflect on the contributions made by the theory of social representations (RS) to understanding the complexity of human responses to human care. For the construction of the document the contributions of classic authors to the theory of RS, these contributions reflect from the experiences of health care were recovered. It identifies possible that this theoretical framework to explain the complexity and variability of human responses of people facing processes of health and disease, and social and historical contexts in which subjects and develop their social group.

Keywords: Nursing, social representations, care.

\section{RESUMEN}

Las repuestas humanas son complejas y dependen de manera relevante de las condiciones sociales, culturales, económicas y políticas de los contextos micro, meso y macro en los que las personas se desenvuelven. El profesional de enfermería requiere desarrollar una perspectiva epistémica, filosófica y metodológica que le posibilite comprender que las maneras en que 
las personas se posicionan frente a los procesos salud-enfermedad no derivan exclusivamente de una decisión personal, sino también de una postura colectivamente construida. El objetivo del ensayo es reflexionar sobre las aportaciones que hace la teoría de las representaciones sociales (RS) a la comprensión de la complejidad de las respuestas humanas frente al cuidado. Para la construcción del ensayo se revisaron las aportaciones de autores clásicos a la teoría de las RS, estos aportes se reflexionan desde experiencias del cuidado de la salud. Se identifica que este marco teórico posibilita explicar la diversa complejidad y variabilidad de las respuestas humanas de las personas frente a los procesos de salud y enfermedad, y de los contextos sociales e históricos en que los sujetos y su grupo social se desarrollan.

Palabras clave: Enfermería, representaciones sociales, cuidados.

\section{RESUMO}

Respostas humanas são complexas e dependem fortemente das condições sociais, culturais, económicos e políticos do micro, meso e macro em que as pessoas vivem contextos. A enfermeira precisa desenvolver uma perspectiva epistemológica, filosófica e metodológica que lhe permite compreender as maneiras pelas quais as pessoas estão posicionados contra processos de saúde-doença não derivam exclusivamente de uma decisão pessoal, mas também uma postura construído coletivamente. O objetivo deste artigo é refletir sobre as contribuições feitas pela teoria das representações sociais (RS) para a compreensão da complexidade das respostas humanas a cuidados humanos. Eles foram revistos contribuições clássicas para a teoria da RS autores refletem suas contribuições são baseadas em experiências de cuidados de saúde. Identificou-se que este quadro teórico permite explicar a complexidade e variabilidade das respostas humanas de pessoas processos de saúde e doença, e contextos sociais e históricos nos quais os indivíduos que enfrentam variados e desenvolver seu grupo social.

Palavras-chave: Enfermagem, representações sociais, cuidados.

\section{INTRODUCCIÓN}

El cuidado es el objeto de estudio de la profesión y debe ser complejizado en el marco biopsicosocial que conforma a sus sujetos de atención, evitando caer en la tentadora evocación de centrar la perspectiva de manera exclusiva en los procesos fisiopatológicos. El profesional de enfermería debe ser consciente que los sujetos se hallan inmersos en contextos sociales, económicos y culturales que al ser diversos, les predisponen esquemas de pensamiento y acción que determinarán las acciones que lleven a cabo para mantener la salud o enfrentar la enfermedad (Hueso, 2006).

La profesión de Enfermería debe hacer frente a los retos que impone una modernidad que impacta en una cada vez mayor diferenciación social, económica y política de las poblaciones a quienes atiende. La desigualdad en derechos, acceso a la educación, salud y empleo, exigen que el profesional de enfermería adopte horizontes teórico-metodológicos que le posibiliten comprender en toda su complejidad, los elementos con que las personas cuentan para potenciar sus procesos de bienestar o confrontar sus procesos de enfermedad.

En el contexto antes descrito es que se considera pertinente reflexionar sobre las aportaciones que hace la teoría de las representaciones sociales (RS) a la comprensión de la complejidad de las respuestas humanas frente 
al cuidado, apostando que tener en consideración el entramado sociocultural en que las personas se desenvuelven potenciará el éxito de las actividades de enfermería tanto asistenciales como de promoción de la salud.

El artículo está organizado en tres apartados que posibilitan comprender la congruencia y relevancia de incorporar nuevos enfoques en la comprensión y la problematización de las respuestas humanas en el cuidado enfermero. El primer segmento abunda en el marco conceptual de las respuestas humanas y la importancia que éstas tienen dentro de las teorías que sustentan actualmente el cuidado; el segundo apartado profundiza sobre el enfoque de las representaciones sociales y su utilidad en la exploración de la subjetividad de los sujetos en el desarrollo de sus respuestas humanas; finalmente, el tercero vincula la pertinencia del enfoque de las representaciones sociales para comprender el tenor en que se construyen las respuestas humanas frente a la salud y la enfermedad.

\section{DESARROLLO DEL TEMA}

\section{Las respuestas humanas como sustento teó-} rico en el cuidado profesional

A principios del siglo XX, la enfermería no se consideraba ni una disciplina académica ni una profesión ${ }^{1}$, fue hasta la aportación de Fawcet de un metaparadigma para la enfermería, que los trabajos de las enfermeras teoristas fueron reconocidos como marcos conceptuales de la profesión. Con la llegada de nuevos marcos y teorías, se planteó una diversidad de perspectivas sobre el paciente y la naturaleza de los cuidados (Marriner, 2003: 14-18).
La enfermería proclama "el cuidado" como objeto de estudio, construyendo respecto al mismo profusos marcos epistémicos, jurídicos, y éticos; con el fin de sustentarlo y consolidar un cuerpo teórico fundamental aplicable al desempeño cotidiano de la disciplina profesional (Daza y Stella, 2006).

La razón de ser de la enfermería es proporcionar cuidados que permitan mantener $y$ conservar la vida, mediante la satisfacción de las necesidades del individuo, familia y comunidad; en función de ello, su campo de acción específico es la predicción, prevención y tratamiento de las respuestas humanas, entendiendo estas como "La forma en que el cliente responde a un estado de salud o enfermedad, representando todos aquellos fenómenos que tienen que ver con el profesional de enfermería” (Federación Mexicana de Colegios de Enfermería, 2013). Estas "formas de responder" se complejizan en una diversidad de percepciones y sentimientos, que de forma subjetiva influyen en la agencia de los sujetos para conservar la salud o limitar el daño en la enfermedad.

Las respuestas humanas han sido descritas como personales y múltiples, su existencia obedece a la naturaleza propia de cada individuo, de la interacción de los sujetos con el entorno y de las experiencias que estos viven en relación con la salud y la enfermedad (Federación Mexicana de Colegios de Enfermería, 2013). Con excepción de las respuestas instintivas, las respuestas humanas no son "naturales", por el contrario, derivan de marcos sociales y culturales en los que prevalecen formas particulares de "ver el mundo" y por tanto, están cargadas de subjetividad.

\footnotetext{
${ }^{1}$ Se considera como disciplina desde el punto de vista académico y hace referencia a una rama de la educación, un departamento educativo o un campo del saber; mientras que el término profesión hace referencia a un campo especializado de trabajo, basado en la estructura teórica de la ciencia o del saber de una disciplina y las habilidades prácticas necesarias para desarrollarlo. Ambos términos entonces se encuentran interrelacionados entre sí.
} 
Durante mucho tiempo, la primera etapa del Proceso de Cuidado Enfermero, denominada "valoración", se focalizó exclusivamente en la identificación de las respuestas fisiopatológicas como resultado de la enfermedad, eso no puede ni debe continuar sucediendo, resulta deseable que el profesional de enfermería desarrolle habilidades para identificar las herramientas con que cuenta la población para confrontar los efectos adversos de la enfermedad o para conservar el bienestar, preocupándose por identificar las necesidades de los pacientes desde una base dialéctica, cognoscitiva y educativa (Izquierdo y Figueroa, 2000).

En el contexto antes descrito, Enfermería debe plantear estrategias para el cuidado individual y colectivo que difieran de las tradicionales, por ejemplo, en el trabajo comunitario, en donde los modelos de gestión deben tener una alta resolución que aseguren la promoción a la salud individual y colectiva (Zarate, 2004)

La Enfermería se sustenta en el humanismo y en función de ello, debe contemplar a sus sujetos de cuidado como personas somato-psíquica-sociales en las que convergen creencias, valores y costumbres estrechamente vinculados con la sociedad y la cultura, variables que condicionan de manera importante su manera de interpretar y actuar frente a la salud y enfermedad (Martínez, 2002)

Desde la premisa anterior, los profesionales de enfermería deben incorporar a sus perspectivas marcos teóricos que les posibiliten analizar los factores que circundan a los sujetos y que son la columna vertebral sobre la que se desarrollan los procesos de salud-enfermedad: El contexto en que se vive, los hábitos e imaginarios que los sujetos conocen y aprenden en los colectivos a que pertenecen respecto al cuidado de la salud, así como los recursos económicos y sociales con que cuentan para llevar a cabo acciones de pro de la salud o en el combate a la enfermedad.

\section{Las representaciones sociales como enfoque de exploración de las subjetividades.}

El concepto de Representación Social (RS), como todo enfoque científico, se ha ido modificando en el curso de su desarrollo, tanto por las reflexiones y contradicciones producidas por su propio uso, como por el impacto de diversos autores en las producciones teóricas y epistemológicas de las ciencias sociales de forma general (González, 2008).

Las RS se aluden como la serie de símbolos y valores que dictan las prácticas que los sujetos comparten dentro del colectivo del que forman parte, no son conscientes y pueden discrepar de las que se expresan verbalmente. Una adolescente puede tener y expresar una noción clara de los riesgos que implica el embarazo a temprana edad, no obstante, desear de forma imperiosa ser madre para no perder a su pareja, para sentirse parte de su comunidad o para ser considerada adulta, y puede buscar la experiencia de la maternidad sin poder explicar en términos verbales que la orilla a tomar esa decisión.

Las RS tienen un compromiso ontológico que no puede ser reducido a los procesos de comunicación contextuales que se producen en un espacio social (grupo social-comunidad), pueden no haberle dicho jamás que si no tiene hijos no será considerada en la toma de decisiones de la comunidad, pero ella observa la diferencia en el trato de una adolescente sin hijos y una madre. Por otra parte, las RS tienen una organización que está más allá de los espacios presentes de relaciones entre las personas y son inseparables de las relaciones que los sujetos desarrollan dentro de un colectivo, es en estas dinámicas donde las RS se transforman 
para adecuarse a los contextos históricos y sociopolíticos específicos.

Aludir el tema de las RS obliga necesariamente a retomar las aportaciones de Michel Foucault, quien profundamente crítico de lo que puede llamarse una concepción tradicional del sujeto, concibe a este como producto del discurso en dos sentidos distintos:

1.- Como un sujeto que personifica las formas particulares de conocimiento que el discurso produce (Aquino, 2013), es decir, donde el sujeto participa en la construcción de símbolos y valores que reproducen las instituciones (familia, iglesia, escuela, etc.), con estos discursos la persona queda "sujeta" a formas particulares de desempeñarse en la realidad.

2.- Como un efecto de estas formaciones discursivas específicas e históricamente situadas (Corvalán, 2005), es decir, mediante los diversos discursos se produce la fabricación de "individuo disciplinario" donde se somete a la persona a un tipo de mentalidad especifica muchas veces lejos de la realidad particular del sujeto y que existe incluso desde antes de su llegada al mundo (Foucault, 2010).

Foucault se enfocó en estudiar cómo se constituyen los sujetos en diferentes momentos y contextos institucionales en tanto objetos de conocimiento posible, deseable e indispensable, y como dentro de este esquema, cobra forma la experiencia de "sí mismos" que son capaces de elaborar en sus particulares contextos. Bajo esta premisa, las experiencias que tienen los sujetos son interpretadas desde el esquema de símbolos y valores que incorporaron desde su llegada al mundo, conformando una identidad que se conserva o se transforma en razón de ciertos fines "socialmente establecidos" y "aceptados".

Las RS son proceso y contenido, como proceso centralizan en la forma en que circulan y se reproducen símbolos y valores socialmente relevantes dentro de un grupo social determinado; como contenido, las RS se ubican en tres dimensiones conocidas como actitud, información y campo de representación.

A continuación se describen los aspectos que consideran cada una de las dimensiones.

1.- La actitud: Constituye el sentido en que las personas regulan sus acciones, ya sea de manera positiva o negativa a los valores establecidos mediante consenso social y en base a una escala de valores colectiva. Es la más evidente de las tres dimensiones.

2.- La información: Se refiere a los conocimientos y la organización de los mismos que posee una persona o grupo sobre un objeto o situación social determinada. No importa sólo la cantidad de información que se tiene, sino la calidad de la misma (prejuicios, mitos, tabús), las fuentes y los intereses particulares de las fuentes desde las que se produce esta información.

3.- El Campo de Representación: Es la manera en que los sujetos seleccionan, priorizan y armonizan las actitudes, opiniones, imágenes, creencias, vivencias y valores presentes en una misma representación social.

En función de lo anterior, las RS sólo pueden comprenderse explorando lo que se sabe (información), lo que se cree y cómo se interpreta (campo de la representación) y la forma en que se lleva a la práctica (actitud).

Las RS posibilitan entender que las acciones del ser humano no dependen únicamente de su experiencia del mundo o de su autonomía, sino derivan de constructos socioculturales que se internalizan convirtiéndose en hábitos que los sujetos asumen en su vida cotidiana.

El papel de las RS en las respuestas humanas frente a los procesos de salud y enfermedad. 


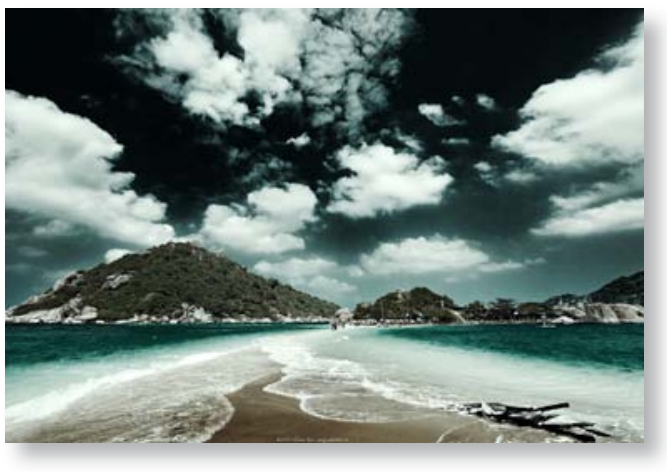

Entender que las respuestas humanas acontecen insertas en contextos socioculturales diversos, obliga a reconocer que los procesos salud-enfermedad sólo pueden comprenderse en la dialógica "individual-social, sujeto-grupo, singularidad-cultura” (Corvalán, 2005).

Las RS que los sujetos poseen respecto a salud, bienestar, cuidado o enfermedad les son transmitidas desde sus etapas iniciales de vida, con la posibilidad de reconfigurarse en función de sus experiencias sociales y los discursos institucionales a que son expuestos.

En el contexto anterior, los sujetos despliegan sus respuestas humanas frente a la salud y enfermedad en virtud de la información que han recibido en contextos socioculturales e históricos específicos. Para Enfermería no bastará hacer llegar la información, deberá considerarse que los sujeto y grupos sociales, elegirán, jerarquizarán y priorizarán la información que reciben en función de los valores que sean más representativos para su grupo social.

Sólo a través del reconocimiento de la premisa anterior es que el profesional de enfermería puede tolerar la frustración de no impactar en el cambio de conductas mediante el discurso, sabiendo que la información que se otorga no es determinante del cambio, dado que esta se integra en un campo de representación en el que los sujetos anclan esta información con otra serie de creencias y valores que son im- portantes para su identidad social y colectiva.

En el tenor anterior, Enfermería debe buscar adecuar el discurso a las creencias y valores que resultan relevantes para el sujeto, proponer estrategias preventivas que sean pertinentes en el marco de la interculturalidad y respetuosas de las cosmovisiones de los colectivos a quienes se dirige el mensaje, potenciando con ello un verdadero empoderamiento de los sujetos para la búsqueda de la salud y el bienestar, o bien para confrontar de manera asertiva los procesos de enfermedad.

La vida cotidiana está cargada de rutinas y símbolos, construidos mediante interacciones grupales en las que se construyen saberes locales que resultan pertinentes a los contextos en que los sujetos se desempeñan socialmente, ello debe ser reconocido en los programas y políticas públicas dirigidas tendientes a disminuir la incidencia de las enfermedades o procurar el bienestar. La ausencia de reconocimiento de la premisa anterior deriva en la falta de éxito de las intervenciones educativas y asistenciales que enfermería lleva a cabo con la población, dado que se pretende que los individuos incorporen de manera literal la información proporcionada, sin reconocer que son sujetos sociales que configuran sus significantes y valores dentro de espacios contextuales en los que se perpetúan conocimientos, creencias y actitudes frente a la salud y enfermedad acordes a un orden social específico (Villegas y González, 2011).

En las repuestas humanas que los sujetos llevan a cabo para mantener la salud o confrontar la enfermedad, existe una amplia diversidad de posturas y capacidades, en función de ello es que se complejiza el cuidado que Enfermería otorga, ya sea en lo preventivo $\mathrm{o}$ asistencial.

Las personas, a pesar de tener una misma enfermedad, reaccionan de formas diversas, 
todo ser humano coloca una huella individual e idiosincrásica sobre la experiencia de la enfermedad, el padecimiento será distinto en función no sólo de la información que haya recibido respecto a su condición, también en función de las creencias e imaginarios que mantenga sobre el dolor. El padecimiento y la patología específica que cursa, se constituye en una construcción colectiva definida por los valores y significantes socialmente construidos en torno a la misma, dejando de ser un ente biológico (o patológico) para constituirse en un constructo cultural definido en término de valores socialmente compartidos por los colectivos.

Esta dualidad sujeto-grupo que se evidencia en las respuestas humanas, está definida por procesos de comunicación que Enfermería debe tomar en cuenta para la construcción de sus discursos de promoción de la salud o autocuidado frente a la enfermedad. Enfermería debe problematizar que en este enfoque dialógico de singularidad-cultura, la reconfiguración de las RS que pueda impulsar mediante sus discursos, marcarán la diferencia para la siguiente generación de colectivos, toda vez que las respuestas humanas están condicionadas por las formas de transmisión de pensamientos, creencias y comportamientos de las personas en la sociedad, son los seres humanos la única especie autoconsciente de sí misma y por ello, capaz de preguntarse y cuestionar su propia realidad (Beorlegui, 2004).

El enfoque de las Representaciones Sociales en las Respuestas humanas posibilita comprender los fenómenos salud-enfermedad desde las perspectivas de las personas, es decir, desde la especificidad de su subjetividad. Una subjetividad que se construye y reconstruye a partir de redes de códigos simbólicos que conforman la cultura partir de su interacción en un mundo social y desde una subjetividad que se contrapone muchas veces a los valores y normas generales en relación a la salud y la enfermedad (Caponi y Goerges, 1997).

Las nuevas tendencias en investigación e innovación del cuidado enfermero, exigen que los profesionales reconozcan las limitaciones de los enfoques positivistas y biomédicos y apuesten por la riqueza de considerar los entornos sociales y culturales en el que intervienen las situaciones de salud y enfermedad (Hueso, 2006).

En el contexto antes planteado resulta necesario reorganizar los sistemas de salud, con el objetivo de superar 2 grandes límites a los cuales se ve enfrentado el hegemónico modelo curativo: a) las interpretaciones mecanicistas ligadas a la bio-medicina y al racionalismo científico, y b) las concepciones reduccionistas y lineales del desarrollo y de la modernidad. Ambos límites tienen consecuencias sobre las definiciones, las estrategias y las políticas de salud para el futuro de nuestras sociedades (Banch, 1990:183-221).

\section{CONCLUSIONES}

La Teoría de las Representaciones Sociales nos permite explicar la diversa complejidad y variabilidad de las respuestas humanas de las personas frente a los procesos de salud y enfermedad, entendiendo que estas respuestas derivan de un marco cultural socialmente constituido en el que se privilegian creencias y valores que se encuentran relevantes para los contextos sociales e históricos en que los sujetos y su grupo social se desarrollan.

Los profesionales de enfermería deben ser sensibles a la identificación de las RS que prevalecen en el contexto de sus sujetos de cuidado, con el fin de construir discursos que contengan información que pueda anclarse con 
facilidad a las RS que los sujetos y grupos históricamente han construido, que no violenten sus creencias/valores y que en consecuencia tengan mayores posibilidades de ser adoptados en sus prácticas cotidianas.

La naturaleza social de los procesos vitales implica realizar un análisis especifico de las particularidades de los contextos de cada uno de los individuos para comprender y actuar en consecuencia a las necesidades reales que permitan lograr efectos positivos de las intervenciones de salud para la promoción y/o atención a la salud.

El comprender cada uno de los contextos en los cuales se encuentran inmersos las personas implicara particularizar las acciones que permitan tener éxito para procurar la salud y bienestar de las personas.

\section{BIBLIOGRAFÍA:}

- Aquino, M.A. (2013). La subjetividad a debate. Sociológica, 28 (80): 259-278. Recuperado de http://www.scielo.org.mx/scielo.php?pid=S0187$\underline{-01732013000300009 \& \text { script }=\text { sci arttext }}$

- Banchs, M.A. (1990). Las representaciones sociales: sugerencia sobre una alternativa teórica y rol posible para los psicólogos sociales en Latinoamérica. México: Universidad de Guadalajara.

- Beorlegui, C. (2004). Antropología filosófica. Nosotros: urdimbre solidaria y responsable. $2^{\text {a }}$ ed. Bilbao: Universidad de Deusto

- Caponi, S., y Goerges, C. (1997). El estatuto epistemológico del concepto de salud. história, ciências, saúde, (2):287-307. Recuperado de http://www.scielo.br/pdf/ hcsm/v4n2/v4n2a05

- Corvalán, F. (2005). 50 Años de Representaciones sociales y Psicología. ECOS 3(1): 115-127. Recuperado de http://www.uff.br/periodicoshumanas/index.php/ecos/ article/viewFile/1055/823

- Daza de Caballero, R., \& Stella, L. (2006). Significado del cuidado de enfermería desde la perspectiva de los profesionales de una institución hospitalaria de tercer nivel en Santa Fe de Bogotá, Colombia. Cultura de los Cuidados, 10(19):55-62. Recuperado de http://rua.ua.es/dspace/ bitstream/10045/968/1/culturacuidados 19 08.pdf

- Federación Mexicana de Colegios de Enfermería (2013). Antología Curso-Taller de Proceso de Enfermería FEMCE. Compilación y actualización. México: Comisión de certificación de Colegios de Enfermería. 14-53. Recuperado de http://www.enfermeriaysaludpublica.edu. $\underline{\mathrm{mx} / 13 / \text { descargas/Antologia2013.pdf }}$

- Foucault, M. (2010). Las palabras y las cosas: Una arqueología de las ciencias humanas. 2a ed. México: Siglo XXI.

- González, R.F. (2008). Subjetividad social, sujeto y representaciones sociales. Diversitas: perspectivas en Psicología 4(2):225-243. Recuperado de http://www.usta. edu.co/otraspaginas/diversitas/doc_pdf/diversitas_8/ vol.4no.2/articulo 1.pdfHueso, M.C. (2006). El padecimiento ante la enfermedad. Un enfoque desde la teoría de la representación social. Índex de enfermería, 15 (55), 49-53. Recuperado de http://scielo.isciii.es/scielo. php?pid $=$ S1132-12962006000300011\&script $=$ sci arttext

- Izquierdo, R.P., Figueroa, C.M. (2000). Diagnósticos de Enfermería identificados en un niño con el síndrome de Denys Gras. Revista cubana de enfermería, 16(13): 185-188. Recuperado de http://scielo.sld.cu/scielo. php?pid $=$ S0864-03192000000300011\&script $=$ sci arttext

- Marriner, T. (2003). Teorías y Modelos de Enfermería: Importancia de la teoría para la enfermería como disciplina y profesión. $5^{\mathrm{a}}$ ed. Madrid: Mosby.

- Martínez, C.F. (2002). Enfermedad y padecer. Ciencia y humanismo en la práctica médica. Historia y filosofía de la medicina. Anales médicos, 47(2), 112-117. Recuperado de http://www.medigraphic.com/pdfs/abc/bc-2002/ bc022k.pdf

- Villegas, M.M., \& González, F.E. (2011) La Investigación Cualitativa de la Vida Cotidiana. Medio Para la Construcción de Conocimiento Sobre lo Social a Partir de lo Individual. Psicoperspectivas, 10(2):35-59. Recuperado de http://www.scielo.cl/pdf/psicop/v10n2/art03.pdf

- Zarate, G.R. (2004). La Gestión del Cuidado de Enfermería. Index de enfermería 13(44-45), 42-46. Recuperado de http://scielo.isciii.es/scielo.php?pid=s113212962004000100009 \&script $=$ sci_arttext 